\title{
Effectiveness of a clinical practice change intervention in increasing the provision of nicotine dependence treatment in inpatient psychiatric facilities: an implementation trial
}

\author{
Paula M. Wye ${ }^{1,2,3}$, Emily A. Stockings ${ }^{4 *}$, Jenny A. Bowman ${ }^{1,2}$, Chris Oldmeadow ${ }^{2}$ and John H. Wiggers $2,3,5$
}

\begin{abstract}
Background: Despite clinical practice guidelines recommending the routine provision of nicotine dependence treatment to smokers in inpatient psychiatric facilities, the prevalence of such treatment provision is low. The aim of this study was to examine the effectiveness of a clinical practice change intervention in increasing clinician recorded provision of nicotine dependence treatment to patients in inpatient psychiatric facilities.

Methods: We undertook an interrupted time series analysis of nicotine dependence treatment provision before, during and after a clinical practice change intervention to increase clinician recorded provision of nicotine dependence treatment for all hospital discharges (aged $>18$ years, $N=4175$ ) over a 19 month period in two inpatient adult psychiatric facilities in New South Wales, Australia. The clinical practice change intervention comprised six key strategies: leadership and consensus, enabling systems and procedures, training and education, information and resources, audit and feedback and an on-site practice change support officer. Systematic medical record audit and segmented logistic regression was used to determine differences in proportions for each nicotine dependence treatment outcome measure between the 'pre', 'during' and 'post-intervention' periods.
\end{abstract}

Results: The prevalence of all five outcome measures increased significantly between the pre and post-intervention periods, including clinician recorded: assessment of patient smoking status (36.43 to 51.95\%; adjusted odds ratio $[A O R]=2.39$, 99\% Confidence Interval [Cl]: 1.23 to 4.66); assessment of patient nicotine dependence status (4.74 to 11.04\%; $\mathrm{AOR}=109.67,99 \% \mathrm{Cl}: 35.35$ to 340.22$)$; provision of brief advice to quit ( 0.85 to $8.81 \%$; $\mathrm{AOR}=97.43,99 \% \mathrm{Cl}$ : 31.03 to 306.30); provision of nicotine replacement therapy ( 8.06 to $26.25 \%$; $\mathrm{AOR}=19.59,99 \% \mathrm{Cl}: 8.17$ to 46.94 ); and provision of nicotine dependence treatment on discharge (8.82 to $13.45 \%, \mathrm{AOR}=12.36$; $99 \% \mathrm{Cl}$ : 6.08 to 25.14 ).

Conclusions: This is the first study to provide evidence that a clinical practice change intervention may increase clinician recorded provision of nicotine dependence treatment in inpatient psychiatric settings. The intervention offers a mechanism for psychiatric facilities to increase the provision of nicotine dependence treatment in accordance with clinical guidelines.

Keywords: Tobacco use disorder, Smoking, Psychiatric department, hospital, Psychiatric nursing, Clinical trial, Interrupted time series analysis, Health plan implementation, Health planning guidelines, Clinical practice nursing research, Healthcare systems

\footnotetext{
* Correspondence: e.stockings@unsw.edu.au

${ }^{4}$ National Drug and Alcohol Research Centre, University of New South Wales,

22-32 King Street, Randwick, New South Wales 2031, Australia

Full list of author information is available at the end of the article
} 


\section{Background}

Smoking remains a leading global cause of death and disability [1]. While smoking rates and the corresponding burden of premature death and disability have declined in the general population, this has not been observed among persons with a mental disorder $[2,3]$. As a consequence, smoking rates are two to three times higher [4], nicotine dependence is more severe [4], quit attempts are less likely to be successful $[5,6]$, and rates of smoking related morbidity and mortality are substantially higher among persons with such a disorder [7, 8]. Smoking prevalence and its' associated burden appear to increase with greater severity of mental illness [9], with higher prevalence among psychiatric inpatients [10]. Reduction of the prevalence of smoking in this population is both a recognised public health priority and a clinical priority for mental health services $[11,12]$.

Despite clinical practice guidelines [13, 14] recommending the provision of nicotine dependence treatment to patients in mental health treatment settings in developed nations such as the United States, United Kingdom and Australia [13-15], the provision of such treatment in psychiatric facilities has been suboptimal, with assessment of nicotine dependence and provision of nicotine replacement therapy (NRT) in inpatient psychiatric facilities occurring rarely $(0 \%-0.5 \%)[16,17]$. Reported barriers to the provision of such treatment include lack of time and clinician skill and experience, and lack of organisational support, even when comprehensive nicotine dependence treatment guidelines are in place [18].

Systematic review evidence supports the effectiveness of a number of clinical practice change strategies for improving health-related practices across a range of clinical settings, including: engaging local opinion leaders [19], audit and feedback [20], reminders [21], clinical decision support systems [22], training and education [23], the development and dissemination of clinical practice guidelines [24], and dissemination of educational materials [22]. Such strategies found to be effective in increasing the implementation of nicotine dependence treatment specifically, include staff training and education [25], and use of electronic health records [26]. A systematic review and meta-analysis of controlled studies demonstrated that such clinical practice change strategies can increase staff provision of assistance to quit to patients in hospital settings (pooled risk difference: 16.6; 95\% confidence interval: 4.9 to 28.3 ) but no effect was found for staff assessment of smoking status, advice to quit, or the provision or discussion of NRT [27]. The review identified no studies that reported the effectiveness of clinical practice change interventions in increasing the implementation of nicotine dependence treatment in inpatient psychiatric facilities.
To address this evidence gap, a study was undertaken to assess the effectiveness of a multi-modal clinical practicechange intervention in increasing clinician recorded provision of five recommended elements of nicotine dependence treatment: 1) assessment of smoking status; 2) assessment of nicotine dependence; 3) provision of brief advice to quit; 4) provision of NRT; and 5) provision of nicotine dependence treatment at discharge to patients admitted to inpatient psychiatric facilities.

\section{Methods \\ Study design}

An interrupted time series study was undertaken. A clinical practice change intervention was implemented for nine months, with outcome data collected for three periods over 19 months: 'pre-intervention' (five months from 1st June to $31^{\text {st }}$ October 2009), 'intervention period' (nine months from $1^{\text {st }}$ November 2009 to $31^{\text {st }}$ July 2010), and 'post-intervention' (five months from $1^{\text {st }}$ August to $31^{\text {st }}$ December 2010).

\section{Setting}

The study was undertaken concurrently in a convenience sample of two of four general locked adult inpatient psychiatric facilities in one health district in New South Wales, Australia. One facility (100 beds) consisted of a discrete psychiatric facility catering for approximately 2000 patient discharges per annum across five units: an emergency/intensive care unit, two general acute units, a dual diagnosis unit, and an older persons unit. The second psychiatric facility (125 beds) catered for approximately 750 patient discharges per annum from a single general acute psychiatric care unit. Both facilities introduced a smoke-free policy in all buildings and grounds three years prior to the study.

\section{Participants}

The study participants consisted of all adult ( $>18$ years) patient discharges, including repeat discharges for individual patients, and for both voluntary and involuntary patients.

\section{Nicotine dependence treatment}

All clinical staff (approximately 170), including registered nurses, nurse managers, allied health professionals, and medical/psychiatric staff received the intervention. Based on both local and international clinical guidelines [13, 14], clinical staff were asked to provide the following five elements of nicotine dependence treatment: 1) assessment of smoking status of all patients on admission; 2) assessment of nicotine dependence; 3) provision of quit advice; 4) provision of NRT (including nicotine patch, lozenge, gum, or inhaler); and 5) provision of any component of nicotine dependence treatment at discharge (including any of brief advice to quit, offer of referral to the Quitline telephone 
support service, and provision of NRT at discharge). Other forms of pharmacotherapies for nicotine dependence treatment, including varenicline and bupropion were not available to patients through the hospital pharmacy, and nicotine oral and nasal sprays were not approved for use in Australia at the time of the study.

\section{Clinical practice change intervention}

The following strategies, informed by clinical practice change evidence [19-24] and evidence of strategies to improve nicotine dependence treatment in healthcare settings [25-27] were implemented to support the routine provision of nicotine dependence treatment by clinical staff:

- Leadership and consensus

a. Consultation with senior medical, nursing and facility management staff

b. Unit level meetings with managers, and clinical leaders and staff using the principles of motivational interviewing [28];

c. Meetings with consumer advocates, and;

d. Appointment of local (minimum of 1 per unit) clinical champions.

- Enabling systems and procedures

a. Distribution of state nicotine dependence treatment guidelines, the NSW Health Guide for the Management of Nicotine Dependent Inpatients [14] (see Additional file 1) to clinical staff, and;

b. Modification of existing admission forms to prompt recording of smoking treatment.

- Training and education

o Face-to-face staff group training sessions $(n=30)$ to increase awareness, agreement and adoption of nicotine dependence treatment guidelines;

o Problem solving with local champions and clinician groups on 'as needs' basis $(n=33)$, and;

o Information sessions for patients and carer groups.

- Practice change information and resources

o All clinical staff and managers were provided: a treatment flowchart (see Additional file 1); a guide for providing and recording treatment; tailored information regarding smoking and mental health and use of NRT; educational resources for patients; and information regarding support for smoking abstinence or cessation by staff.

- Audit and feedback

o Audit and feedback of unit medical records regarding prevalence of nicotine dependence treatment $(n=14$ face to face information sessions held in total with clinical staff across the six units to provide tailored feedback on the performance of their unit).

\section{- Practice change Support}

A practice change support officer (0.5 FTE) was available to support delivery of the above strategies.

\section{Data collection procedures}

All components of the medical record for each patient discharged during the study period were audited postdischarge by trained auditors employed by the research team $[16,29]$. Auditors were non-clinical health service staff based in the hospital medical records department, were not specifically linked to or affiliated with the psychiatric facilities and were independent of the study team. Auditors were aware that the audit pertained to tobacco use, but were not privy to the intervention activities.

\section{Measures}

\section{a) Patient characteristics}

The following patient characteristics were obtained from the medical record: gender, age (determined by date of birth), admission and discharge dates, previous admissions (yes, no), number of discharges during study period), treating unit at discharge, Aboriginal or Torres Strait Islander status (yes, no, unknown), English speaking (yes, no, unknown). Patient diagnosis was classified via ICD-10 codes [30].

b) Nicotine dependence treatment

Based on the NSW Health Guide for the Management of Nicotine Dependent Inpatients [14], see Additional file 1) the recorded provision of five elements of nicotine dependence treatment was audited:

1. assessment of smoking status (yes, no);

2. assessment of nicotine dependence (yes, no);

3. provision of brief advice to quit (yes, no);

4. prescription of any form of NRT (including nicotine patch, lozenge, gum, or inhaler) (yes, no), and;

5. provision of nicotine dependence treatment on discharge record (any of: provision of written information/brief advice to quit, offer of referral to Quitline, and provision of NRT post discharge) (yes, no).

\section{Analysis}

Analyses were conducted using SAS version 9.4 software [31]. Admission and discharge dates were used to calculate length of stay. Diagnoses were collapsed into eight most prevalent categories: schizophrenia/psychosis; affective disorders; adjustment/anxiety disorders; substance use disorders; borderline personality disorder; bipolar disorder; 
dementias, and other mental disorder. For smoking status assessment and provision of brief advice outcomes, audit data from multiple medical record locations were collapsed to form a single measure (recorded/not recorded anywhere).

Descriptive statistics were used to summarise patient characteristics, and prevalence of each of the nicotine dependence treatment measures at each of three time periods (pre-intervention, intervention and postintervention). Chi-square analyses were undertaken to assess differences in patient socio-demographic and clinical characteristics between patients with one or more admission, and between study periods.

Similar to other studies of nicotine dependence treatment in hospital settings based on medical record audit $[32,33]$, each outcome measure is reported as a proportion of the total patient sample (both smokers and nonsmokers). Smoking prevalence was assumed to be constant during the study period, based on previous research conducted in this facility [16, 34], and elsewhere [2]. The proportions for each outcome were plotted for each fortnight in the study period [35]. Segmented logistic regression analysis of interrupted time series data was used to determine differences in outcome proportions between the pre- and during, during and post-, and pre to post-intervention periods, to plot outcome proportions across the 19-month study period [36]. Month was the unit of time chosen to generate the slopes in the logistic regression analyses, and as such there were five time points (i.e. months) in the preintervention period (1st June to $31^{\text {st }}$ October 2009), nine time points in the 'intervention period' $\left(1^{\text {st }}\right.$ November 2009 to $31^{\text {st }}$ July 2010), and five time points in the 'postintervention' period ( $1^{\text {st }}$ August to $31^{\text {st }}$ December 2010). The models adjusted for differences in patient sociodemographic and clinical characteristics (including discharging unit) between study periods. All discharges were treated independently in the analyses. All statistical tests were two-tailed, and the threshold for statistical significance was $p<0.01$. We used the conservative $1 \%$ threshold for statistical significance (and corresponding 99\% Confidence Intervals [CIs]) in an attempt to correct for an increased overall type I error rate from the multiple tests (and intervals) that were conducted.

\section{Results}

\section{Patient characteristics}

There were 4,175 discharges (1054 pre-intervention; 2054 during intervention and 1078 post-intervention) during the 19 month study period for 2,898 individual patients. Nearly one third of total discharges $(n=1277$, $30.6 \%$ ) were for patients readmitted to the facilities during the study period. There were no differences in socio-demographic or clinical characteristics between patients who had one or multiple admissions.
Across the total study period, patients were mostly male (55\%), had an average age of 39.2 years $(\mathrm{SD}=15.3)$ and an average length of stay of 17.0 days $(\mathrm{SD}=33.4)$. The most prevalent primary diagnoses on admission were schizophrenia and related psychosis (25\%), adjustment disorders $(18 \%)$ and unipolar affective disorders (17\%). Primary diagnosis on admission, and discharging unit were identified to vary significantly between study periods $(p<.0001)$, and these variable were added as confounding variables to the segmented logistic regression models (Table 1).

\section{Nicotine dependence treatment}

The following results were robust to adjustment for potentially confounding variables, and did not differ between patients who had one or multiple admissions. Adjusted results are shown in text, and unadjusted results are provided in Table 1 in the Additional file 1.

\section{Assessment of smoking status}

The prevalence of recorded assessment of patient smoking status increased significantly between each of the study periods, with an overall significant increase from pre- to post-intervention (15.52\% absolute increase, 99\% Confidence Interval [CI]: 10.04 to 20.99; Table 2). As shown in Fig. 1 and Table 3, overall, the odds of smoking status being assessed increased significantly from pre-intervention to the end of the postintervention period (adjusted odds ratio $[\mathrm{AOR}]=2.39$, 99\% CI: 1.23 to 4.66 ), driven by increases from the preto intervention period (Fig. 1, Table 3).

\section{Assessment of nicotine dependence}

The prevalence of recorded assessment of nicotine dependence was stable in the pre-intervention phase, and increased significantly from intervention to postintervention, with an overall significant increase from preto post-intervention $(6.30 \%$ absolute increase, $99 \% \mathrm{CI}$ : 3.31 to 9.28; Table 2). As shown in Fig. 1 and Table 3, overall the odds of a nicotine dependence assessment being recorded increased significantly from pre-intervention to the end of the post-intervention period ( $\mathrm{AOR}=109.67$, 99\% CI 35.35, 340.22; Table 3), with increases in odds occurring between each time period (Fig. 1, Table 3).

\section{Provision of brief advice to quit}

The prevalence of recorded brief advice to quit increased significantly between each of the study periods, with an overall significant increase from pre-to post intervention $(7.96 \%$ absolute increase, $99 \%$ CI: 5.62 to 10.30; Table 2). As shown in Fig. 1 and Table 3, the odds of recorded provision of brief advice to quit increased 
Table 1 Socio-demographic and clinical characteristics of the patient sample at the three audit periods, pre- $(n=1054)$, during $(n=2043)$ and post-intervention $(n=1078)$

\begin{tabular}{|c|c|c|c|c|c|}
\hline \multirow[b]{2}{*}{ Variable } & \multicolumn{4}{|l|}{ Time period } & \multirow[b]{2}{*}{$P$} \\
\hline & $\begin{array}{l}\text { Pre intervention } \\
(n=1054)\end{array}$ & $\begin{array}{l}\text { Intervention period } \\
(n=2043)\end{array}$ & $\begin{array}{l}\text { Post intervention } \\
(n=1078)\end{array}$ & $\begin{array}{l}\text { Total sample } \\
(n=4175)\end{array}$ & \\
\hline Gender & & & & & 0.8397 \\
\hline Male & $573(54 \%)$ & $1110(54 \%)$ & $597(55 \%)$ & $2280(55 \%)$ & \\
\hline First admission to facility? & & & & & 0.1123 \\
\hline Yes & $410(39 \%)$ & $841(41 \%)$ & $402(38 \%)$ & $1653(40 \%)$ & \\
\hline Planned discharge? & & & & & 0.3193 \\
\hline Yes & 1019 (99\%) & $2004(99 \%)$ & 1064 (99\%) & 4087(99\%) & \\
\hline Discharging unit & & & & & $<.0001$ \\
\hline Emergency and intensive care & $93(9 \%)$ & $364(18 \%)$ & $241(23 \%)$ & $698(17 \%)$ & \\
\hline General acute unit 1 & $235(23 \%)$ & $334(16 \%)$ & $152(14 \%)$ & $721(17 \%)$ & \\
\hline General acute unit 2 & $226(22 \%)$ & $465(23 \%)$ & $164(15 \%)$ & $855(20 \%)$ & \\
\hline Comorbid mental health and substance use unit & $209(20 \%)$ & $341(17 \%)$ & 207 (19\%) & $757(18 \%)$ & \\
\hline Rural acute unit & $223(21 \%)$ & $443(22 \%)$ & $243(23 \%)$ & $909(22 \%)$ & \\
\hline Older persons unit & $52(5 \%)$ & $89(4 \%)$ & $66(6 \%)$ & $207(5 \%)$ & \\
\hline Identified as Aboriginal or Torres Strait Islander? & & & & & 0.0325 \\
\hline Yes & $83(7.9 \%)$ & $199(9.8 \%)$ & $121(11 \%)$ & $403(10 \%)$ & \\
\hline No or unknown & $968(92 \%)$ & $1840(90 \%)$ & $955(89 \%)$ & $3763(90 \%)$ & \\
\hline English speaking & & & & & 0.0232 \\
\hline Yes & 1019 (97\%) & $1994(98 \%)$ & $1062(99 \%)$ & 4075(98\%) & \\
\hline No or unknown & $32(3.0 \%)$ & $46(2.3 \%)$ & $14(1.3 \%)$ & & \\
\hline Primary diagnosis on admission & & & & & $<0.0001$ \\
\hline Schizophrenia and related psychosis & $262(25 \%)$ & $524(26 \%)$ & $251(23 \%)$ & $1037(25 \%)$ & \\
\hline Unipolar affective disorder & $204(19 \%)$ & $320(16 \%)$ & $170(16 \%)$ & $694(17 \%)$ & \\
\hline Bipolar disorder & $91(8.7 \%)$ & $204(10 \%)$ & $108(10 \%)$ & $403(10 \%)$ & \\
\hline Adjustment disorder & $181(17 \%)$ & $355(17 \%)$ & $201(19 \%)$ & $737(18 \%)$ & \\
\hline Borderline personality disorders & $104(10 \%)$ & $190(9 \%)$ & $74(7 \%)$ & $368(9 \%)$ & \\
\hline Other mental disorder & $40(4 \%)$ & $60(3 \%)$ & $34(3 \%)$ & 134(3\%) & \\
\hline Drug use disorders & $118(11 \%)$ & 277 (14\%) & $198(18 \%)$ & $593(14 \%)$ & \\
\hline Dementias & $13(1.2 \%)$ & $12(0.6 \%)$ & $5(0.5 \%)$ & $30(1 \%)$ & \\
\hline Other medical condition & $34(3 \%)$ & $93(5 \%)$ & $36(3 \%)$ & 163(4\%) & \\
\hline Age in years & & & & & 0.1345 \\
\hline mean (SD) & $39.0(15.5)$ & $38.9(15.0)$ & $40.0(15.5)$ & $39.2(15.3)$ & \\
\hline Length of stay & & & & & 0.5144 \\
\hline mean (SD) & $16.1(35.9)$ & $17.6(34.4)$ & $16.7(28.4)$ & $17.0(33.4)$ & \\
\hline
\end{tabular}

significantly from pre to post-intervention $(A O R=97.43$, 99\% CI 31.03, 306.30), with increases in odds occurring between each time period (Table 3).

\section{Prescription of NRT}

The prevalence of recorded prescription of NRT increased significantly between each of the study periods, with an overall significant increase from pre-to post- intervention (18.19\% absolute increase, $99 \%$ CI: 14.12 to 22.26; Table 2). As shown in Fig. 1 and Table 3, the odds of recorded prescription of NRT increased significantly from pre- to post-intervention $(\mathrm{AOR}=19.59,99 \% \mathrm{CI}$ : 8.17 to 46.94 ), with this being driven by increases from the pre- to intervention period (Table 3).

5. Provision of nicotine dependence treatment at discharge 


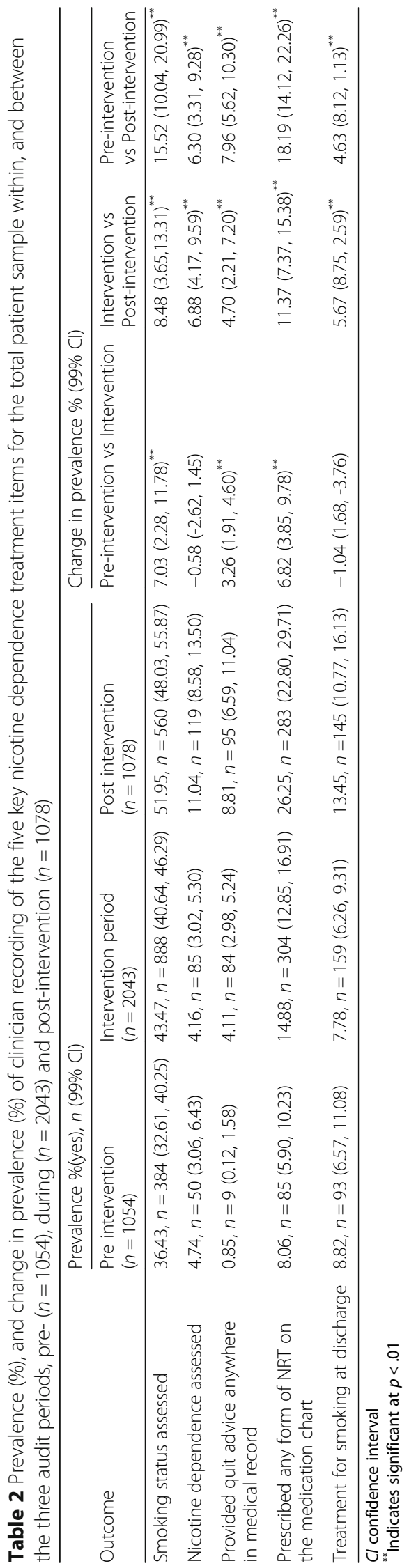




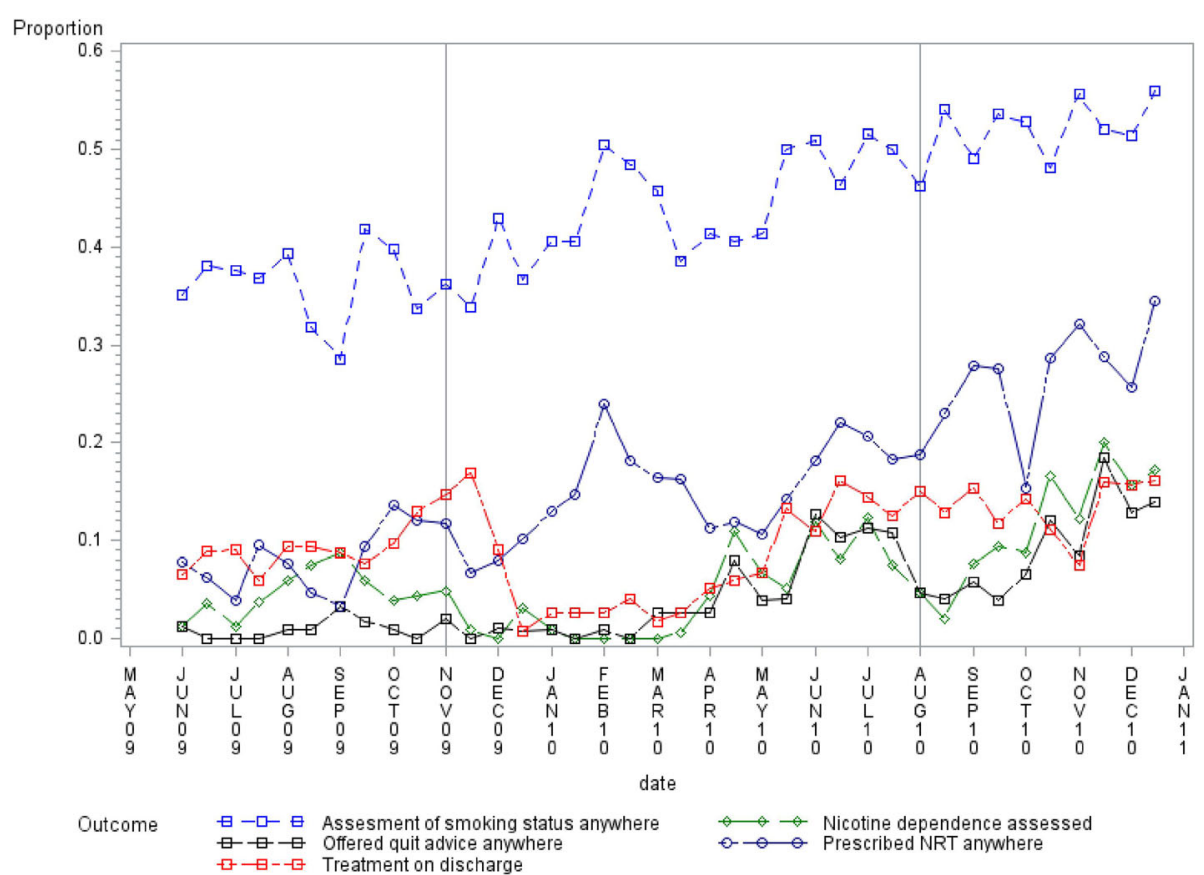

Fig. 1 The proportions (yes) of each of the five nicotine dependence treatment outcomes, plotted fortnightly for the total patient sample at pre$\left(1^{\text {st }}\right.$ June-31 $1^{\text {st }}$ October, 2009, $\left.n=1054\right)$, during $\left(1^{\text {st }}\right.$ November $2009-31^{\text {st }}$ July 2010, $\left.n=2043\right)$ and post-intervention $\left(1^{\text {st }}\right.$ August- $31^{\text {st }}$ December 2010, $n=1078)$

The prevalence of recorded provision of nicotine dependence treatment on the discharge summary was stable from pre- to during-intervention, but increased significantly from during to post-intervention, with an overall significant increase from pre- to postintervention $(4.63 \%$ absolute increase, $99 \% \mathrm{CI}: 1.13$ to 8.12). As shown in Fig. 1 and Table 3, the odds of recorded provision of nicotine dependence treatment at discharge increased significantly from pre- to postintervention ( $\mathrm{AOR}=12.36,99 \% \mathrm{CI}$ : 6.08 to 25.14$)$, with this being driven by increases from the intervention to post-intervention period (Table 3).

Stratified results for the 100-bed ( 2000 discharges per annum) and 125-bed facility ( 750 discharges per annum) are shown in Tables 2 and 3 of the Additional file 1 . The direction and strength of the associations in the two facilities was largely similar to the overall aggregated findings. However, due to the lower number of annual admissions in the 125-bed facility, the number of events was small and power was reduced, resulting in wide confidence intervals around the estimates for this facility (see Additional file 1: Table 3).

\section{Discussion}

This is the first study to examine the effectiveness of a clinical practice change intervention in increasing the recorded provision of nicotine dependence treatment in inpatient psychiatric facilities. The findings suggest that the intervention was effective in increasing clinicianrecorded provision of nicotine dependence treatment, with increases identified for all five nicotine dependence treatment measures during the intervention period, and continuing for up to five months post-intervention.

The magnitude of the observed improvement in recording of nicotine dependence treatment in our study are similar to [18], and in some cases greater than [37] those reported in previous studies conducted in general hospital facilities. For example, a four-year interrupted time series study utilising a similar multi-component clinical practice change intervention in 37 general public hospitals in Australia [38] found significant increases in recorded provision of nicotine dependence treatment from pre to post-intervention, including: advice to quit (8.6\% increase vs. $8 \%$ in our study); and provision of inpatient NRT (16\% vs. $18 \%$ ) [38]. Similarly a controlled study conducted across four hospitals in the same state as the current study identified increases in nicotine dependence treatment that ranged from 9 to $22 \%$ [39]. It is particularly encouraging that in the present study, the increases in nicotine dependence treatment occurred in inpatient psychiatric settings-settings known to have higher rates of patient smoking [10], and negative staff attitudes to delivering smoking treatment [40].

The observed rates of recorded provision of the five nicotine dependence treatment variables were stable across the pre-intervention period, and increased in 


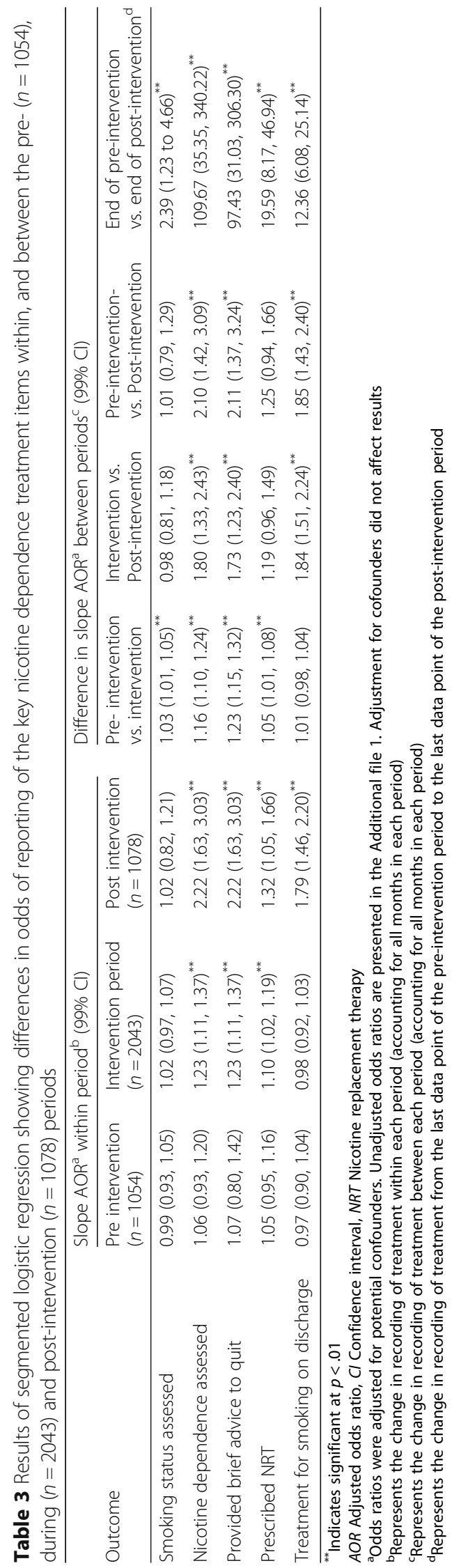


accordance with the timing of the delivery of the clinical practice change intervention. Critically, these increases continued into the post-intervention period, suggesting not only the efficacy of the clinical practice change intervention, but some degree of sustainability of effect. This finding adds to the growing body of evidence that multimodal nicotine dependence treatment interventions show efficacy in increasing nicotine dependence treatment provision in hospital settings both during, and after implementation of the intervention [38]. Further, the observed increases across multiple elements of nicotine dependence treatment are noteworthy, as previous studies have indicated improvements in some but not all forms of treatment assessed [27, 38]. This finding is particularly important, as there is evidence that patient receipt of multiple elements of such treatment increases the likelihood of stopping smoking [13, 41, 42]. It is possible that the lower baseline proportions of patients receiving nicotine dependence treatment in our study (e.g. $0.85 \%$ for quit advice) compared to general medical settings (e.g. 5.4\% for quit advice in Slattery et al.'s [38] study), paired with the higher rates of smoking and nicotine dependence in these samples relative to the general population $[3,10]$ meant that there was greater scope for improvement to be made and detected across all nicotine dependence outcomes in the current study. Further, there are inherent differences across studies in intervention length, intensity, follow-up length and outcome measurement that may have differentially influenced the results across these studies, the degree to which is unknown.

Notwithstanding the consistent increases in all outcomes, at post-intervention, an assessment of smoking status occurred for only $52 \%$ of patients, with even lower rates for other elements of nicotine dependence treatment. This suggests that additional strategies may be required to ensure that all patients receive nicotine dependence treatment. Previous research has indicated that interventions that are integrated with, and address existing hospital systems and procedures may maximise nicotine dependence treatment delivery [13, 32, 39, 43]. For example, enhancing hospital accountability by including nicotine dependence treatment indicators in hospital accreditation and performance processes has been suggested to increase the provision of such care [44, 45]. Similarly, incorporating nicotine dependence treatment within existing hospital computer systems (such as an electronic medical record system) has been shown to increase smoking care, and may also reduce clinician time burden [32]. The improved, but still suboptimal rates of smoking status assessment and nicotine dependence treatment at post-intervention in this study may have been due to the absence of such strategies in the intervention, and/or the length of the intervention period. Little research has investigated the intervention strategy intensity or duration required for successful guideline implementation and clinical practice change $[19,20]$. Similarly, due to the complex, multi-modal and interrelated nature of the intervention components used in the current study, we are unable to comment on which single component-or combinations of components-had the greatest effect, which may be important for implementation in clinical settings where resources may be limited. Future researchers in this area may consider examining the effect of individual intervention components and collecting data regarding intervention implementation costs, in order to advise cost-effectiveness analyses.

This study has multiple strengths, including its' large sample size, inclusion of a number of patient diagnostic groups, and use of systematically independently collected medical record audit data. However, a number of study characteristics may have impacted on its findings. Firstly, we relied on medical record audit as a measure of clinical staff provision of nicotine dependence treatment. It is possible that clinical staff may have recorded care delivery in the absence of it occurring, and hence overestimated actual delivery of nicotine dependence treatment. However, systematic review evidence suggests that medical record audit is more likely to underestimate clinical staff behaviour [46]. Although the audit staff were non-clinical health service staff who were independent of the research team and were not privy to the intervention activities, they may have become aware of the intervention process via being part of the broader health system. Thus we cannot exclude the possibility that the audit staff may have recorded data more rigorously in the post-intervention period than before. Further, although the hospital nicotine dependence treatment guidelines recommend a clear 'pathway' of care (see Additional file 1) where smoking status is assessed, brief advice is provided, NRT is prescribed and patients are monitored until discharge, it is unclear from the audit data to what extent clinical staff followed this pathway in a linear matter. For example, NRT may have been provided before (or in the absence of) provision of brief advice to quit, particularly so for patients who may be well known to clinical staff due to repeat admissions. While it is out of scope here to comment on each individual patient's nicotine dependence treatment pathway, future studies in this area may aim to examine both global increases care delivery (as demonstrated here), but also improvements in adherence to the care pathway as detailed in clinical practice guidelines.

Secondly, given that the recording of smoking status did not occur systematically for all patients at any time point during the study, the true sample of smokers for whom nicotine dependence treatment should be delivered was unknown. Previous research conducted among 
patients in the same facility within the same timeframe indicated that smoking rates were approximately 53\% $[47,48]$. Hence, the rates of nicotine dependence treatment presented here are likely to underestimate the true proportion of smokers receiving nicotine dependence treatment. Nevertheless, assuming that the prevalence of smoking status remained stable across the study period $[32,33]$, the observed increases in nicotine dependence treatment provision over time are considered to likely reflect an increase in nicotine dependence treatment delivery to patients who are smokers.

Third, given our focus on increasing clinicianrecorded nicotine dependence treatment according to the standard hospital care guidelines, we did not collect data on other factors that may have had an impact on the likelihood of clinical staff providing and recording such treatment to patients. This may have included the voluntary or involuntary status of patients, which has shown to be important in the implementation of smokefree policies in locked psychiatric facilities, given the relative abilities of these patients to leave the grounds to smoke. While we did not collect such data in our study, in Australia in 2014-15 it was estimated that $31.1 \%$ of all hospitalisations to specialised psychiatric care services were for patients who had an involuntary admission [49]. These two groups may represent distinct clinical cohorts for whom there may have been a differential effect of the intervention [50]. We also did not collect data regarding patient use of other pharmacological treatments for smoking cessation outside of those provided as part of standard hospital care guidelines, including varenicline and bupropion (which were not available through the hospital pharmacy) or oral or nasal nicotine spray (which was not approved for use in Australia at the time of the study). The use of such other pharmacotherapies outside of those provided as standard hospital care may have influenced the likelihood of clinicians providing routine hospital nicotine dependence treatment to patients.

Further, utilising a controlled trial design was not feasible, and the use of a non-controlled design constrains our ability to directly attribute the observed increases in recording of nicotine dependence treatment to the intervention component. Given the limited resources available, the study facilities were required to be within close geographical proximity to the administering University, and were too dissimilar in size and annual patient admissions in order for one site to act as a comparator to the other. As such, the interrupted time series design was adopted, as it has been demonstrated to be appropriate in evaluating the efficacy of clinical practice change interventions in hospital settings where wholeof-system practice change is the focus of the intervention [38]. Further, this pragmatic study design provides one means of accelerating the research translation process by simultaneously disseminating the intervention strategy whilst conducting the evaluation [51]. Where feasible, future researchers aiming to examine the efficacy of a clinical practice change intervention should consider the inclusion of a comparison condition, such as through the use of a randomised controlled trial design (e.g. [52]) or through hybrid evaluation designs, such multiple baseline (e.g. [53]) or stepped wedge cluster randomised designs [51].

Finally, although the study data were collected from 2009-2010, more recent evaluations of the study facilities by the authorship team indicate that prevalence of smoking among patients, and clinician provision of nicotine dependence treatment (including both patient report and medical record audit) are unlikely to have changed in this time [34, 47, 54]. Given that this study is the first to examine the efficacy of a clinical practice change intervention in increasing nicotine dependence treatment to smokers within inpatient psychiatric facilities, it provides a strong basis for future studies further develop the intervention design and outcome measures, and to explore potential differential effects of the intervention among specific subgroups of the patient population, including those with voluntary/involuntary admission status, and those who may be using other pharmacotherapies, such as varenicline and bupropion.

\section{Conclusions}

This study is the first to examine the effectiveness of a multi-modal clinical practice change intervention in increasing recorded nicotine dependence treatment in inpatient psychiatric facilities. The use of inpatient psychiatric facilities as a setting for supporting and encouraging smokers to change behaviour may make an important contribution to addressing the recognised need to reduce smoking rates for this population group. Our results provide evidence that improvement in the routine recording of nicotine dependence treatment is achievable on a whole-of hospital basis and are sustainable in the short to medium term. However additional strategies are required to ensure all smokers are systematically identified and offered nicotine dependence treatment.

\section{Additional file}

Additional file 1: (DOCX $228 \mathrm{~kb})$

\section{Abbreviations}

Cl: Confidence interval; FTE: Full time equivalent; ICD: International Classification of Diseases; NRT: Nicotine replacement therapy; OR: Odds ratio; SD: Standard deviation 


\section{Acknowledgements}

We would like to acknowledge the assistance of the staff and patients of the Hunter New England Mental Health inpatient services for their involvement in the trial.

\section{Funding}

PW is supported by an NHMRC/PHCRED Early Career Fellowship (\#1037241). ES is supported by an NHMRC Early Career Fellowship (\#1104600). The research was jointly funded by the NHMRC National Institute of Clinical Studies/ Hospitals Contribution Fund, the Hunter Medical Research Institute; and the Australian Primary Health Care Research Institute. None of these funders had any role in the conceptualisation or conduct of this study.

\section{Availability of data and materials}

Requests for raw data and supporting materials can be made by contacting the corresponding author.

\section{Authors' contributions}

PW, JB and JW contributed to the research design, and designed the audit tool, and PW led the implementation of the intervention component. ES, PW, JB and JW led the development of this manuscript. CO developed the statistical analysis plan and undertook the analyses. All authors contributed to, read, and approved the final version of this manuscript. The corresponding author confirms that she had full access to all the data in the study and had final responsibility for the decision to submit the manuscript for publication.

\section{Competing interests}

The authors declare that they have no competing interest.

\section{Consent to publish}

Not applicable.

\section{Ethics approval and consent to participate}

Ethics approval was obtained from the Hunter New England Human Research Ethics Committee (Approval No. 09/03/18/4.04) and the University of Newcastle Human Research Ethics Committee (Approval No. H-2010-0007).

\section{Author details}

'School of Psychology, University of Newcastle, University Drive, Callaghan, New South Wales 2308, Australia. ${ }^{2}$ Hunter Medical Research Institute, Lot 1 Kookaburra Circuit, New Lambton Heights, New South Wales 2305, Australia. ${ }^{3}$ Hunter New England Population Health (HNEPH), Longworth Ave, Wallsend, New South Wales 2287, Australia. ${ }^{4}$ National Drug and Alcohol Research Centre, University of New South Wales, 22-32 King Street, Randwick, New South Wales 2031, Australia. ${ }^{5}$ School of Medicine and Public Health, University of Newcastle, University Drive, Callaghan, New South Wales 2308, Australia.

Received: 21 June 2016 Accepted: 31 January 2017

Published online: 07 February 2017

\section{References}

1. Lim SS, et al. A comparative risk assessment of burden of disease and injury attributable to 67 risk factors and risk factor clusters in 21 regions, 19902010: a systematic analysis for the Global Burden of Disease Study 2010. Lancet. 2012;380(9859):2224-60.

2. Cook B, et al. Trends in smoking among adults with mental illness and association between mental health treatment and smoking cessation. JAMA. 2014;311(2):172-82.

3. Britton J. Treating smoking in mental health settings. Lancet Psychiatry. 2015;2(5):364-5.

4. Australian Institute of Health and Welfare. National drug strategy household survey: detailed findings. Canberra: AlHW; 2007.

5. Diaz F, et al. Datapoints: smoking and smoking cessation among persons with severe mental illnesses. Psychiatr Serv. 2006;57(4):462.

6. Hagman B, et al. Tobacco use among those with serious psychological distress: results from the national survey of drug use and health. Addict Behav. 2008;33(4):582-92.

7. Jones DR, et al. Prevalence, severity, and co-occurrence of chronic physical health problems of persons with serious mental illness. Psychiatr Serv. 2004; 55(11):1250-7.
8. Davidson S, et al. The general health status of people with mental illness. Australas Psychiatry. 2001;8(1):31-5.

9. Farrell $\mathrm{M}$, et al. Nicotine, alcohol and drug dependence and psychiatric comorbidity. Results of a national household survey. Br J Psychiatry. 2001; 179:432-7.

10. Lineberry TW, et al. Population-based prevalence of smoking in psychiatric inpatients: a focus on acute suicide risk and major diagnostic groups. Compr Psychiatry. 2009;50(6):526-32.

11. Royal College of Physicians and Royal College of Psychiatrists. Smoking and mental health. London: RCP Council Report CR178; 2013.

12. Williams JM, Willett JG, Miller G. Partnership between tobacco control programs and offices of mental health needed to reduce smoking rates in the United States. JAMA Psychiat. 2013;70(12):1261-2.

13. Fiore M, Jaen C, Baker T. Treating tobacco use and dependence: 2008 update: clinical practice guideline. Rockville: U.S. Department of Health and Human Services: Public Health Service; 2008

14. New South Wales Department of Health. Guide for the management of nicotine dependent inpatients. Sydney: State Government of New South Wales; 2002.

15. Ratschen E, Britton J, McNeill A. Implementation of smoke-free policies in mental health in-patient settings in England. Br J Psychiatry. 2009;194(6):547-51.

16. Wye $\mathrm{P}$, et al. An audit of the prevalence of recorded nicotine dependence treatment in an Australian psychiatric hospital. Aust N Z J Public Health. 2010;34(3):298-303.

17. Prochaska JJ, Gill P, Hall SM. Treatment of tobacco use in an inpatient psychiatric setting. Psychiatr Serv. 2004;55(11):1265-70.

18. Freund $\mathrm{M}$, et al. Smoking care provision in hospitals: a review of prevalence. Nicotine Tob Res. 2008;10(5):757-74.

19. Flodgren $\mathrm{G}$, et al. Local opinion leaders: effects on professional practice and health care outcomes. Cochrane Database Syst Rev. 2011;8:CD000125.

20. Ivers $\mathrm{N}$, et al. Audit and feedback: effects on professional practice and healthcare outcomes. Cochrane Database Syst Rev. 2012;6:CD000259.

21. Grimshaw J, et al. Toward evidence-based quality improvement. Evidence (and its limitations) of the effectiveness of guideline dissemination and implementation strategies 1966-1998. JOurnal of General Internal Medicine. 2006;21 Suppl 2:S14-20.

22. Grimshaw JM, et al. Effectiveness and efficiency of guideline dissemination and implementation strategies. Health Technol Assess. 2004;8(6):iii-iv. 1-72.

23. Forsetlund $L$, et al. Continuing education meetings and workshops: effects on professional practice and health care outcomes. Cochrane Database Syst Rev. 2009;2:CD003030.

24. Giguere A, et al. Printed educational materials: effects on professional practice and healthcare outcomes. Cochrane Database Syst Rev. 2012;10:CD004398.

25. Carson KV, et al. Training health professionals in smoking cessation. The Cochrane Database of Systematic Reviews. 2012;5:Cd000214.

26. Boyle R, Solberg L, Fiore M. Use of electronic health records to support smoking cessation. The Cochrane Database of Systematic Reviews. 2014;12:Cd008743.

27. Freund $M$, et al. Increasing smoking cessation care provision in hospitals: a meta-analysis of intervention effect. Nicotine Tob Res. 2009;11(6):650-62.

28. Burke BL, Arkowitz H, Menchola M. The efficacy of motivational interviewing: a meta-analysis of controlled clinical trials. J Consult Clin Psychol. 2003;71(5):843-61.

29. Shaw C, Costain D. Guidelines for medical audit: seven principles. Br Med J. 1989:299(6697):498-9.

30. World Health Organisation. The ICD-10 classification of mental and behavioural disorders: clinical descriptions and diagnostic guidelines. Geneva: World Health Organization; 1992.

31. SAS Institute Inc. The SAS System for Windows, Release version 9.4. Cary: SAS Institute; 2013.

32. Koplan KE, et al. A Computerized Aid to Support Smoking Cessation Treatment for Hospital Patients. J Gen Intern Med. 2008;23(8):1214-7.

33. Chang HC, Zimmerman LH, Beck JM. Impact of chart reminders on smoking cessation practices of pulmonary physicians. Am J Respir Crit Care Med. 1995;152(3):984-7.

34. Wye $P$, et al. Observation of the extent of smoking in a mental health inpatient facility with a smoke-free policy. BMC Psychiatry. 2014;14(1):94.

35. Jandoc R, et al. Interrupted time series analysis in drug utilization research is increasing: systematic review and recommendations. J Clin Epidemiol. 2015;68(8):950-6.

36. Penfold RB, Zhang F. Use of Interrupted Time Series Analysis in Evaluating Health Care Quality Improvements. Acad Pediatr. 2013;13(6, Supplement): S38-44. 
37. Katz DA, et al. Implementing Smoking Cessation Guidelines for Hospitalized Veterans: Effects on Nurse Attitudes and Performance. J Gen Intern Med. 2013;28(11):1420-9.

38. Slattery C, et al. Increasing smoking cessation care across a network of hospitals: an implementation study. Implement Sci. 2016;11(1):28.

39. Freund $\mathrm{M}$, et al. Increasing hospital-wide delivery of smoking cessation care for nicotine-dependent in-patients: a multi-strategic intervention trial. Addiction. 2009;104:839-49.

40. McNally $L$, et al. A survey of staff attitudes to smoking-related policy and intervention in psychiatric and general health care settings. J Public Health. 2006;28(3):192-6.

41. Fiore MC, Goplerud E, Schroeder SA. The Joint Commission's New Tobacco Cessation Measures - Will Hospitals Do the Right Thing? N Engl J Med. 2012;366(13):1172-4.

42. National Institute for Health and Care Excellence (NICE). Smoking cessation in secondary care: acute, maternity and mental heath services. London: NICE; 2013.

43. Vidrine Jl, et al. Ask-Advise-Connect: a new approach to smoking treatment delivery in health care settings. JAMA Intern Med. 2013;173(6):458-64.

44. Chassin MR, et al. Accountability Measures - Using Measurement to Promote Quality Improvement. N Engl J Med. 2010;363(7):683-8.

45. Schmaltz SP, et al. Hospital performance trends on national quality measures and the association with joint commission accreditation. J Hosp Med. 2011;6(8):454-61.

46. Hrisos $\mathrm{S}$, et al. Are there valid proxy measures of clinical behaviour? a systematic review. Implement Sci. 2009;4(1):37.

47. Stockings EA, et al. Implementation of a smoke-free policy in an inpatient psychiatric facility: Patient-reported adherence, support, and receipt of nicotine-dependence treatment. Int J Ment Health Nurs. 2015;24(4):342-9.

48. Stockings EA, et al. Impact of a postdischarge smoking cessation intervention for smokers admitted to an inpatient psychiatric facility: a randomized controlled trial. Nicotine Tob Res. 2014;16(11):1417-28.

49. Australian Institute of Health and Welfare, Mental health services-in brief. 2016, AlHW. Cat. no. HSE 180: Canberra

50. Campion J, et al. Implementing smoke-free policies in mental health inpatient units: learning from unsuccessful experience. Australas Psychiatry. 2008;16(2):92-7.

51. Wolfenden $\mathrm{L}$, et al. Improving the translation of health promotion interventions using effectiveness-implementation hybrid designs in program evaluations. Health Promot J Austr. 2016;27(3):204-7.

52. Cooke M, Mattick R, Walsh RA. Implementation of the 'Fresh Start' smoking cessation programme to 23 antenatal clinics: A randomized controlled trial investigating two methods of dissemination. Drug Alcohol Rev. 2001;96:495-505.

53. Bartlem KM, et al. Effectiveness of an intervention in increasing the provision of preventive care by community mental health services: a non-randomized, multiple baseline implementation trial. Implement Sci. 2016;11(1):46.

54. Metse AP, et al. Evaluating the efficacy of an integrated smoking cessation intervention for mental health patients: study protocol for a randomised controlled trial. Trials. 2014;15:266

\section{Submit your next manuscript to BioMed Central and we will help you at every step:}

- We accept pre-submission inquiries

- Our selector tool helps you to find the most relevant journal

- We provide round the clock customer support

- Convenient online submission

- Thorough peer review

- Inclusion in PubMed and all major indexing services

- Maximum visibility for your research

Submit your manuscript at www.biomedcentral.com/submit

) Biomed Central 